\title{
The Natural Plant Product Rottlerin Activates Kv7.1/KCNE1 Channels
}

\author{
Veronika Matschkea,b Ilaria Piccinib-e Janina Schubert ${ }^{b}$ Eva Wrobel ${ }^{b}$ Florian Lang ${ }^{f}$ \\ Johann Matschke ${ }^{g}$ Elsie Amedonu ${ }^{\text {b,h }}$ Sven G. Meuth ${ }^{h}$ Timo Strünker \\ Nathalie Strutz-Seebohm ${ }^{b}$ Boris Greberc,d Jürgen Scherkenbeck ${ }^{j}$ \\ Guiscard Seebohm be
}

aDepartment of Cytology, Institute of Anatomy, Ruhr-University Bochum, Bochum, 'DInstitute for Genetics of Heart Diseases (IfGH), Department of Cardiovascular Medicine, University Hospital Muenster, Muenster, 'Human Stem Cell Pluripotency Laboratory, Max Planck Institute for Molecular Biomedicine, Muenster, ${ }^{d}$ Chemical Genomics Centre of the Max Planck Society, Dortmund, eInterdisziplinäres Zentrum für Klinische Forschung (IMF) Muenster, ${ }^{f}$ Department of Cardiology, Vascular Medicine and Physiology, University of Tuebingen, Tuebingen, IInstitute of Cell Biology, University Hospital Essen, Essen, ${ }^{h}$ Department of Neurology, University of Muenster, Muenster, ${ }^{1}$ Centre of Reproductive Medicine and Andrology, University Hospital Muenster, Muenster, jUniversity of Wuppertal, Faculty of Chemistry, Wuppertal, Germany

\section{Key Words}

$\mathrm{KCNQ} \cdot$ Activator $\bullet$ Mallotoxin $\bullet \mathrm{K}$ channel opener $\bullet$ Cardiac $\bullet$ Heart $\bullet$ iPSC

\begin{abstract}
Background/Aims: Acquired as well as inherited channelopathies are disorders that are caused by altered ion channel function. A family of channels whose malfunction is associated with different channelopathies is the $\mathrm{Kv7} \mathrm{K}^{+}$channel family; and restoration of normal Kv7 channel function by small molecule modulators is a promising approach for treatment of these often fatal diseases. Methods: Here, we show the modulation of Kv7 channels by the natural compound Rottlerin heterologously expressed in Xenopus laevis oocytes and on iPSC cardiomyocytes overexpressing Kv7.1 channels. Results: We show that currents carried by Kv7.1 $\left(\mathrm{EC}_{50}=1.48 \mu \mathrm{M}\right), \mathrm{Kv} 7.1 / \mathrm{KCNE} 1\left(\mathrm{EC}_{50}=4.9 \mu \mathrm{M}\right)$, and Kv7.4 $\left(\mathrm{EC}_{50}=0.148 \mu \mathrm{M}\right)$ are strongly enhanced by the compound, whereas Kv7.2, Kv7.2/Kv7.3, and Kv7.5 are not sensitive to Rottlerin. Studies on Kv7.1/KCNE1 mutants and in silico modelling indicate that Rottlerin binds to the R-L3-activator site. Rottlerin mediated activation of Kv7.1/KCNE1 channels might be a promising approach in long QT syndrome. As a proof of concept, we show that Rottlerin shortens cardiac repolarisation in iPSC-derived cardiomyocytes expressing Kv7.1.Conclusion: Rottlerin or an optimized derivative holds a potential as QT interval correcting drug.
\end{abstract}




\section{Cellular Physiology Cell Physiol Biochem 2016;40:1549-1558

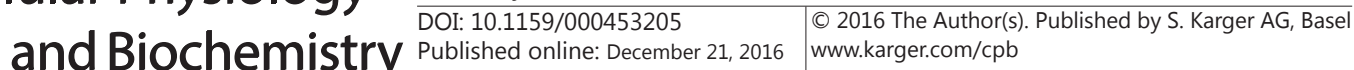 \\ Matschke et al.: Rottlerin Modulates Kv7 Channels}

\section{Introduction}

Ion channels control the specificity, timing, and extend of ion passage across cellular membranes. Cation-selective channels control several physiological processes such as the membrane potential and excitability of single cells and tissues, ion homeostasis, cell volume, vesicle trafficking, as well as neurotransmitter and hormone secretion. Therefore, it is not surprising that dysfunction of cation channels results in a variety of pathophysiological conditions. $\mathrm{K}^{+}$-selective channels are the genetically and functionally most diverse family of cation channels, and many therapeutically relevant drugs mediate their effects by targeting $\mathrm{K}^{+}$channels [1].

Members of the voltage-gated delayed rectifier Kv7 family of $\mathrm{K}^{+}$channels are expressed in brain, heart, thyroid gland, pancreas, inner ear, muscle, stomach and intestine. These channel proteins are required for maintenance of diverse body functions [2]. Mutations in four out of five $K C N Q$ genes (KCNQ1-5) that encode for $\mathrm{K}_{\mathrm{v}} 7.1-\mathrm{K}_{\mathrm{v}} 7.5$ have been associated with inherited channelopathies. Mutations in KCNQ1 cause cardiac arrhythmia in long QT (LQT) or short QT (SQT) syndromes with or without deafness [3, 4] and epilepsy [5]. The channels may be involved in diabetes [6, 7] and thyroid gland disorders [8,9]. KCNQ2 and KCNQ3 mutations cause benign familial neonatal epilepsy (BFNS) [10-12]. Finally, mutations in $K C N Q 4$ underlie congenital deafness and possibly hypertension $[13,14]$. Consistently, all known disease-causing mutations in Kv7.1-4 alter the function of the respective channel isoform [14-18], leading to changes in membrane potential and excitability. However, no disease has so far been directly related to mutations in the KCNQ5 gene and Kv1.5 channel, respectively. Because Kv7 channelopathies result from altered Kv7 channel function, pharmacological targeting of $\mathrm{K}_{\mathrm{v}} 7$ channels represents an attractive approach for treatment of the diseases. Particularly, activation of $\mathrm{K}_{\mathrm{v}} 7$ channels by derivatives of channel agonists like retigabine, R-L3, NH29, Tanshinone IIA, hyperchlorphene, PBA, ML277, and BMS-204352 may be useful for treatment of hypertension, cardiac arrhythmia, impaired hearing, epilepsy, and incontinence. In 1998, the first highly potent and selective Kv7.1 activator, R-L3, was developed [19]. R-L3 is a gating modifier that decreases the rate of channel deactivation and, moreover, increases macroscopic current amplitudes by a mechanism independent of the effect on the gating kinetics [20]. R-L3 shortens action potential duration and suppresses early afterdepolarizations in ventricular myocytes isolated from hypertrophied rabbit hearts. Based on a drug-induced LQT model, R-L3 can be expected to be beneficial in forms of iLQTS and aLQTS [21]. Further, R-L3 activates most of the LQTS-associated mutant channels [20]. However, one Kv7.1 mutation is reported to disrupt R-L3 binding and, thereby, channel activation [20]. Recently, further Kv7.1 activators have been described [22-25]. Potent activators of Kv7.4 include BMS-204352 [26], but often lack selectivity. In the heart and the inner ear, Kv7.1 and Kv7.4 channels may associate with the ß-subunits KCNE1-5 [27] that modulate the drug sensitivity of heteromeric Kv7/KCNE channel complexes [20, 28, 29].

Here, we report the activation of heterologously expressed Kv7.1, Kv7.1/KCNE1 and Kv7.4 channels by Rottlerin. Rottlerin - also known as Mallotoxin - is a naturally occurring chemical that is obtained from the flower dust of the capsule fruit of the tree Mallotus phillippinensis. Mallotoxin was patented in 2006 for the therapy of hypertension (patent WO/2006/060196). Finally, we show that Rottlerin-mediated activation of Kv7.1/KCNE1 in iPSC-derived cardiomyocytes alters cardiac repolarisation.

\section{Materials and Methods}

Molecular Biology

Molecular biological procedures were performed as described before [27, 29, 30]. Briefly; human Kv7.1 (NM_000218.2, variant 1), human KCNE1 (NM_000219.3), rat Kv7.2 (AF087453), human Kv7.3 (AF033347), human Kv7.4 (AF105202) and human Kv7.5 (AF249278) were subcloned into the oocyte expression vectors pSGEM, pTLN, pSP64 or pXOOM. The vectors were digested using adequate restriction enzymes 


\section{Cellular Physiology Cell Physiol Biochem 2016;40:1549-1558 \begin{tabular}{l|l|l} 
and Biochemistry Published online: December 21,2016 & $\begin{array}{l}\text { (c) } 2016 \text { The Author(s). Published by S. Karger AG, Basel } \\
\text { www.karger.com/cpb }\end{array}$
\end{tabular} \\ Matschke et al.: Rottlerin Modulates Kv7 Channels}

for linearization and in-vitro synthesis of cRNA was performed with SP6 or T7 mMessage mMachine kit (Thermo Fisher, Darmstadt, Germany).

\section{Electrophysiology}

Stage V Xenopus laevis oocytes were obtained by EcoCyte Bioscience (Castrop-Rauxel, Germany). Oocyte handling was performed as previously described [27]. The oocytes were injected with $40-60 \mathrm{nl}$ of cRNA solution. Oocytes were injected with 4 ng of Kv7.x cRNA alone or with 3 ng Kv7.1 plus 3 ng of KCNE1 cRNA. The oocytes were stored for 3 - 4 days at $17^{\circ} \mathrm{C}$ in Barth's solution (88 mM NaCl, $1.1 \mathrm{mM} \mathrm{KCl,} 2.4 \mathrm{mM}$ $\mathrm{NaHCO}_{3}, 300 \mu \mathrm{M} \mathrm{Ca}\left(\mathrm{NO}_{3}\right)_{2}, 300 \mu \mathrm{M} \mathrm{CaCl}_{2}, 800 \mu \mathrm{M} \mathrm{MgSO}_{4}, 15 \mathrm{mM}$ HEPES-NaOH, Streptomycin sulfate (20 $\mathrm{mg} / \mathrm{l}$ ), Penicillin-G (31 mg/l) and Gentamycin (50 mg/l), pH 7.6). Standard TEVC recordings were performed at about $22^{\circ} \mathrm{C}$ using a Turbo Tec-10CX amplifier (NPI electronic, Tamm, Germany) linked by a Digidata 1322A interface to an IBM compatible computer running the software pCLAMP 8.0 (Axon Instruments Inc./ Molecular Devices, Sunnyvale CA, USA). Recordings were analyzed using software packages ClampFit 8.0 (Axon Instruments Inc. / Molecular Devices, Sunnyvale CA, USA) and Origin 7.0 (Additive, Friedrichsdorf, Germany). Recording pipettes were filled with $3 \mathrm{M} \mathrm{KCl}$ and had resistances of 0.5 - $1.5 \mathrm{M} \Omega$. Potassium channel currents were recorded in ND96 recording solution $(96 \mathrm{mM} \mathrm{NaCl}, 4 \mathrm{mM} \mathrm{KCl}, 1.8 \mathrm{mM} \mathrm{MgCl} 2,1.0$ $\mathrm{mM} \mathrm{CaCl}{ }_{2}, 5 \mathrm{mM} \mathrm{HEPES}$; pH 7.6). Rottlerin was purchased from Enzo life sciences (Cat. No. ALX-350-075, Lörrach, Germany). All other reagents where obtained from Sigma-Aldrich (Steinheim, Germany). Rottlerin was freshly prepared before every experiment from a $1 \mathrm{mM}$ or $10 \mathrm{mM}$ DMSO stock solution. Control Patch Clamp recordings were done using automated Patch Clamp using the Patch-liner (Nanion).

\section{IPSC derived cardiomyocytes}

Generation and handling of Kv7.1 over-expressing cardiomyocytes have been described before [31]. Here, we utilized the identical protocol and 2 weeks old beating Kv7.1-EGFP expressing cardiomyocytes. Cardiomyocytes were plated on MEA chips. Rottlerin $(2 \mu \mathrm{M}$ or $5 \mu \mathrm{M})$ was directly applied and the signal of several electrodes was averaged to generate ECG-like field potential recordings as described before [31].

\section{Data analysis}

The current-voltage relationship was analyzed at the end of the depolarization. Current amplitudes were plotted vs. the respective depolarizing voltage. The conductance/voltage curves were constructed by plotting the initial (determined $\sim 5 \mathrm{~ms}$ after the end of the test pulse) normalized tail current amplitudes $\left(\mathrm{I}_{\text {tail }}\right)$ versus the respective test potential. These curves were fitted to a Boltzmann equation of the form:

$I_{\text {tail }}=\left[A_{1}-A_{2} / 1+\exp \left(\left(V_{1 / 2}-V\right) / k\right)\right]+A_{2}$

$V_{1 / 2}$ is the voltage of half-maximal activation; $k$ is the slope factor; $V$ is the voltage of the test pulse; $A_{1}$ and $\mathrm{A}_{2}$ are the maximal and minimal current amplitudes respectively.

Data were tested for significance using Student's t-test and ANOVA, and results with $\mathrm{p}<0.05$ were considered statistically significant.

\section{Results}

Rottlerin activates Kv7.1, Kv7.1/KCNE1 and Kv7.4

We studied the action of Rottlerin on heterologously expressed Kv7 channels. To this end, we expressed Kv7.1, Kv7.2, Kv7.2/Kv7.3, Kv7.4, and Kv7.5 in Xenopus laevis oocytes, which gave rise to characteristic currents (Fig. 1B). Rottlerin (30 $\mu \mathrm{M})$ enhanced outward currents (at $40 \mathrm{mV}$ ) carried by Kv7.1 and Kv7.4 by $30.8+/-3.16 \%$ and $29.4+/-3.38 \%$, respectively (Fig. 1B, C). In contrast, currents carried by Kv7.2/7.3 heteromers, Kv7.2, and Kv7.5 were similar in the absence and presence of Rottlerin. In the heart and the inner ear (stria vascularis), Kv7.1 forms heteromeric channel complexes with KCNE1 to conduct the native $\mathrm{I}_{\mathrm{Ks}}$. KCNE1 modulates the pharmacology of Kv7.1 [29]. Therefore, we tested the action of Rottlerin on Kv7.1/KCNE1 heteromers. Currents carried by Kv7.1/KCNE1 were enhanced by $33+/-2.24 \%$, demonstrating the sensitivity to Rottlerin is preserved in the heteromeric channel (Fig. 1B, C). The drug enhanced the currents in a dose-dependent fashion (Fig. 2), and analysis of the dose-response relation using the Hill equation yielded concentrations of half maximal activation $\left(\mathrm{EC}_{50}\right)$ of $1.48 \mu \mathrm{M}, 4.8 \mu \mathrm{M}$, and $0.148 \mu \mathrm{M}$ for its action on $\mathrm{Kv7.1}$, KARGER 


\section{Cellular Physiology Cell Physiol Biochem 2016;40:1549-1558

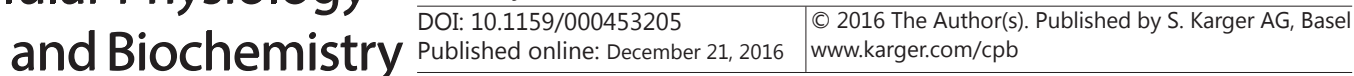 \\ Matschke et al.: Rottlerin Modulates Kv7 Channels}

Kv7.1/KCNE1, and Kv7.4, respectively.

Rottlerin shifts the voltage dependence of activation in Kv7.1 and Kv7.1/KCNE1 but not Kv7.4

Kv7 channel activators evoke various kinetic responses, depending on the class of activator. In order to analyse the mechanism of Rottlerin action, we analysed I/V and G/V relationships in the absence and presence of Rottlerin. In Kv7.1 and Kv7.1/KCNE1, Rottlerin evoked a considerable left-shift of the voltage-dependence of activation by $-19.3 \mathrm{mV}$ and $-10.5 \mathrm{mV}$; respectively (Fig. 3, Table 1). In contrast, Rottlerin did not shift the voltage-dependence of Kv7.4, indicating a different mode of action (Fig. 3, Table 1).

Rottlerin may bind to Kv7.1/KCNE1 channels at the R-L3 site

In order to obtain deeper insight into the potentiation of Kv7.1/KCNE1 currents by Rottlerin, we tested whether the drug might also reduce endocytosis and, thereby, increase the number of channels in the plasma membrane (Fig. 4A). We utilized a mutant Kv7.1 ${ }^{\mathrm{APY}}$ channel that cannot be ubiquitinylated and, thus, escapes ubiquitinguided endocytosis [32]. In oocytes expressing the mutant channel, Rottlerin still enhanced currents amplitudes, arguing against that the drug enhances the surface expression (Fig. 4A). Next, we tested whether the drug binds directly to the channel protein. Residue Kv7.1 $1^{\text {Ile } 37}$ is positioned in the centre of the R-L3 activator binding site [33], and Kv7.1 $1^{1337 v}$ mutant channels are almost insensitive to this Kv7.1 activator. Of note, the Kv7.1 $1^{1337 v}$ mutant was insensitive to Rottlerin, indicating that Rottlerin interacts with the R-L3 binding site.

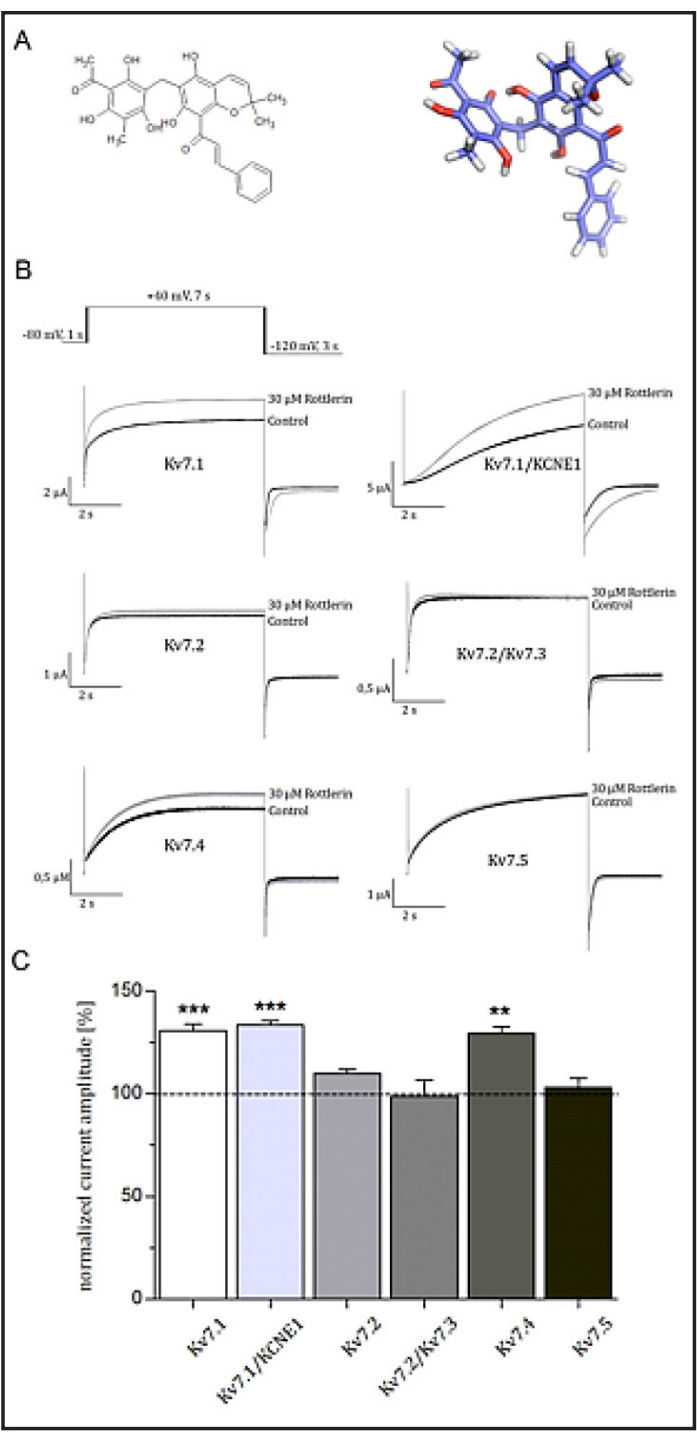

Fig. 1. Action of Rottlerin on Kv7 channels. A Structure of Rottlerin. $B, C$ Effect of Rottlerin on Kv7 currents, determined by repetitive pulses to $+40 \mathrm{mV}$ from a holding potential of $-80 \mathrm{mV}$. During an initial control period oocyte was perfused with bath solution containing $0.3 \%$ DMSO followed by perfusion with $30 \mu \mathrm{M}$ drug containing solution ( $\mathrm{n}=8-19, \pm$ SEM, unpaired t-test $\left.{ }^{* *} \mathrm{p}<0,001,{ }^{* * *} \mathrm{p}<0,0001\right)$.

Rottlerin preferentially binds in silico between voltage sensors and the pore domain of Kv7.1 channels

To support this notion, we performed molecular modelling and Rottlerin docking. Rottlerin molecules preferably bind between the Kv7.1 voltage sensors and the outer pore domain, a region overlapping with the R-L3 binding site [33]. To further support the hypothesis of direct Rottlerin binding to the channel, we performed in silico simulations. Automatic docking of Rottlerin to a Kv7.1 and Kv7.1/KCNE1 open channel model using an indexed version of software Autodock identifies several putative binding sites which are exclusively localized outside the pore domain in proximity of the voltage sensor domain (Fig. 4B). The virtually highest affine docking position for Rottlerin was located at a crevice between voltage sensors and the pore domain (S5-S6 outer face). 
A
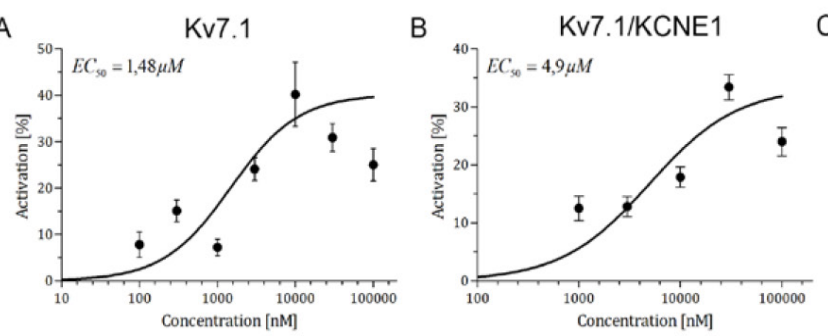

Fig. 2. Concentration dependence of Rottlerin action. Dose-response relationship for Rottlerin activation of Kv7.1 (A), Kv7.1/KCNE1 (B) and Kv7.4 (C) channel current ( $\mathrm{n}=8-17, \pm$ SEM). The resultant stimulation was plotted vs the respective Rottlerin concentrations. The data were fitted to a Hill equation, yielding the concentrations of half maximal activation.

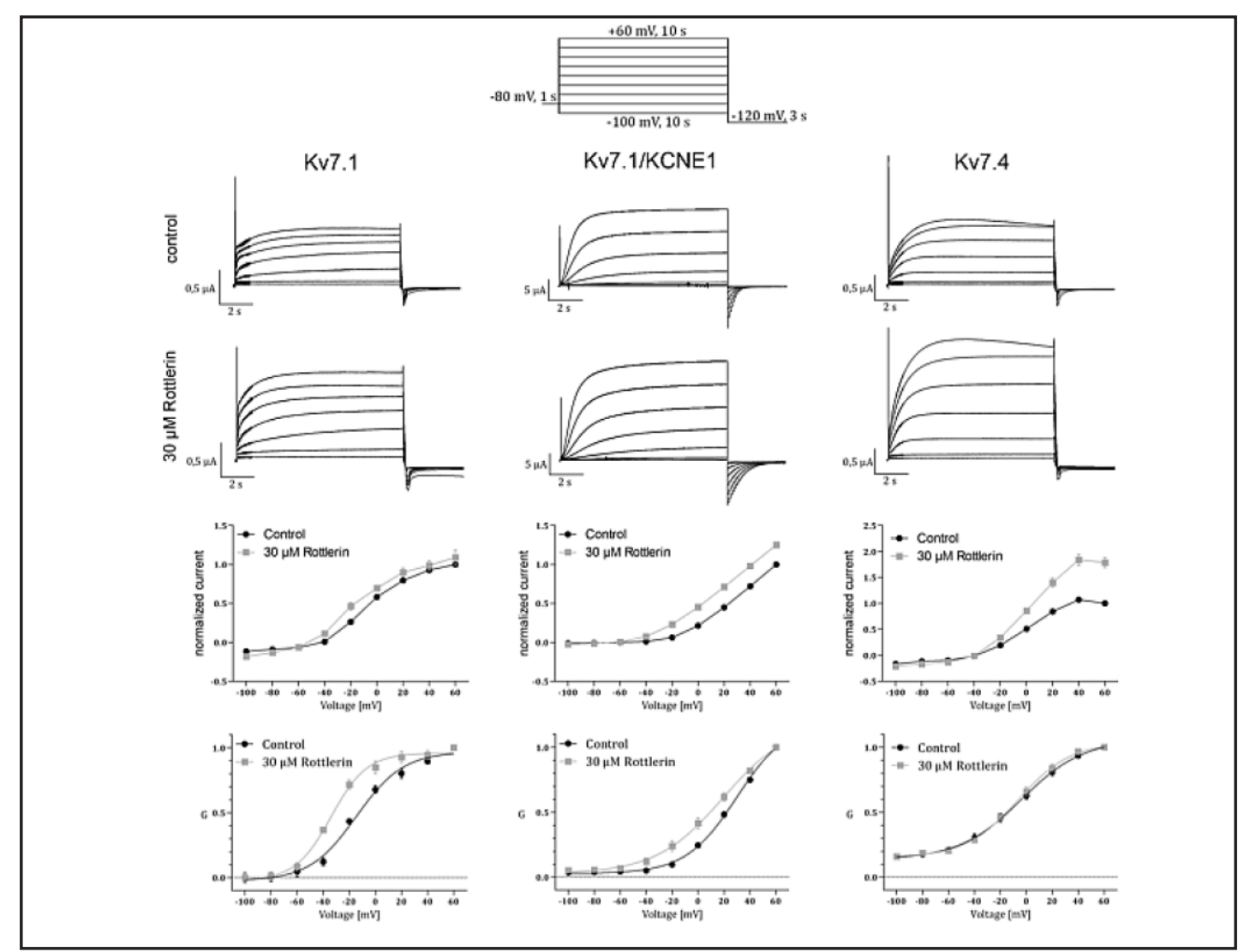

Fig. 3. Activation of Kv7.1 and Kv7.1/KCNE1 by Rottlerin is associated with altered voltage dependence of activation. Current-voltage relationship and voltage-dependence of activation determined from peak tail currents at $-120 \mathrm{mV}$. Values were normalized to peak currents at $+60 \mathrm{mV}$ of Kv7.1 in the absence and presence of of $30 \mu \mathrm{M}$ Rottlerin $(\mathrm{n}=6, \pm \mathrm{SEM})$. Current-voltage relationship and voltage-dependence of activation determined from peak tail currents at $-120 \mathrm{mV}$. Values were normalized to peak currents at $+60 \mathrm{mV}$ of Kv7.1/KCNE1 in the absence and presence of $30 \mu \mathrm{M}$ Rottlerin $(\mathrm{n}=8, \pm \mathrm{SEM})$. Current-voltage relationship and voltage-dependence of activation determined from peak tail currents at $-120 \mathrm{mV}$. Values were normalized to peak currents at $+60 \mathrm{mV}$ of Kv7.4 with and without application of $30 \mu \mathrm{M}$ Rottlerin $(\mathrm{n}=4, \pm \mathrm{SEM})$.

Table 1. Half maximal activation voltage $\mathrm{V}_{1 / 2}$ of the homomeric Kv7.1 and Kv7.4, and the heteromeric Kv7.1/KCNE1 chan-

\begin{tabular}{llll}
\hline & Kv7.1 & Kv7.1/KCNE1 & Kv7.4 \\
\hline $\mathrm{V}_{1 / 2}$ control $(\mathrm{mV})$ & $-14.5 \pm 2.3$ & $30.0 \pm 2.4$ & $-3.5 \pm 2.7$ \\
\hline
\end{tabular}
nel in the absence (cont
ence of Rottlerin $(30 \mu \mathrm{M})$ 


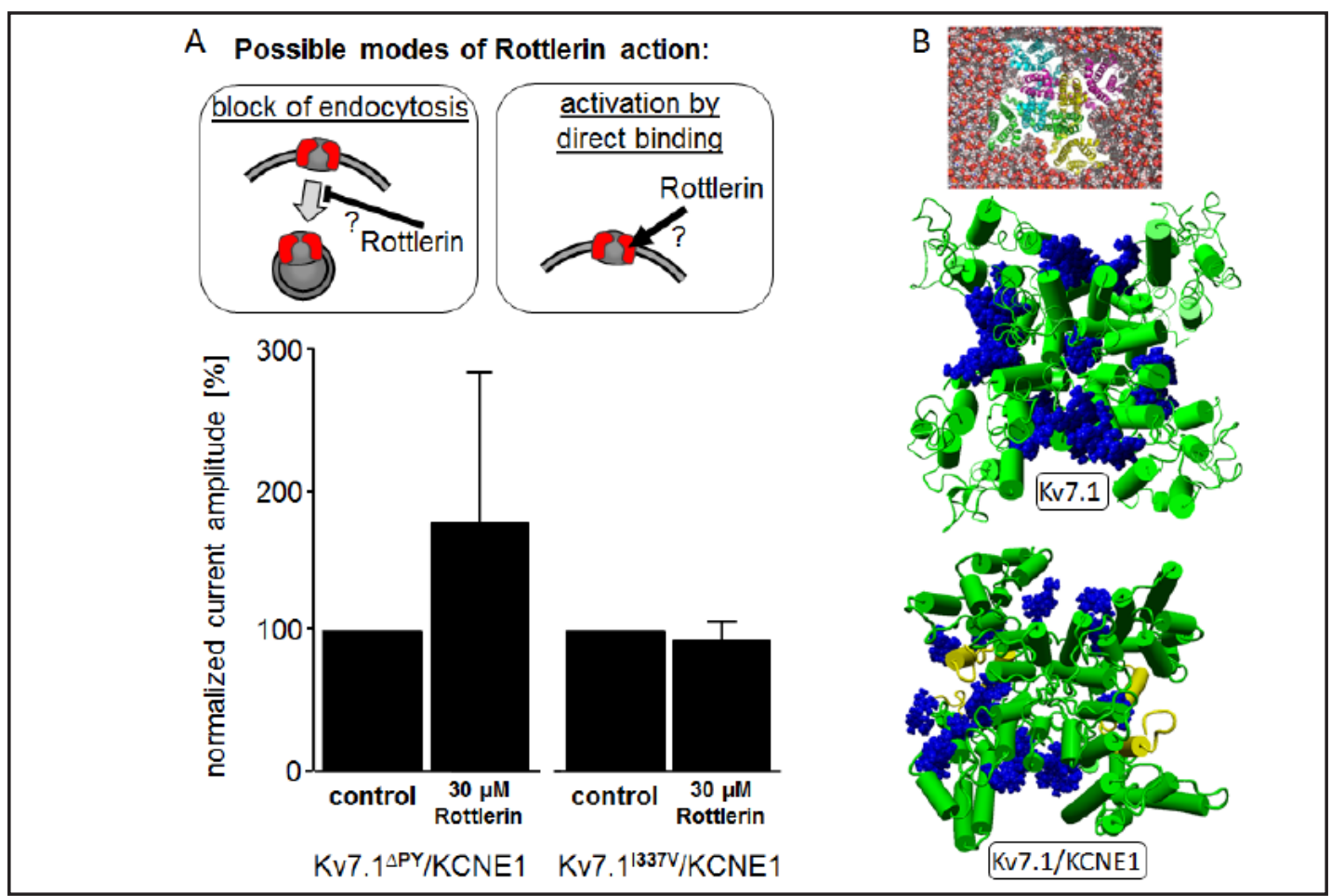

Fig. 4. Analysis of alternative modes of Rottlerin action. A. Rottlerin-stimulation of Kv7.1/KCNE1 can result from block of channel endocytosis leading to increased channels at the plasma membrane and function. Alternatively, direct binding of Rottlerin can activate channels. Mutant channels Kv7.1 ${ }^{\triangle \mathrm{PY}} / \mathrm{KCNE} 1$ and Kv7.1 $1^{1337 V} /$ KCNE1 were expressed in oocytes and their Rottlerin tested by the identical pulse protocol as depicted in Figure 1B. Rottlerin activates mutant Kv7.1 $1^{\triangle \mathrm{PY}} / \mathrm{KCNE} 1$ channels that have disabled ubiquitinguided endocytosis whereas mutant Kv7.1 1337v /KCNE1 channels with disrupted R-L3 binding site are Rottlerin insensitive. $B$. Homology modeling and docking techniques were performed with YASARA Structure 10.1. A consensus homology model of Kv7.1 was generated from a Kv2.1/Kv1.2-chimera (pdb: 2R9R). A refinement with defined solvent molecules (33\% phosphatidylcholine, $33 \%$ Phosphatidylserine, $33 \%$ phosphatidylethanolamine, pH 7.0, $298 \mathrm{~K}$, solvent: $0.9 \% \mathrm{NaCl}$ solvent density 0.997 , force field AMBER03, coulomb electrostatics at a cutoff 7.86) and an Autodock docking-simulation was run. $B$ Top view of the extracellular membrane. Lipid molecules are colored in CPK color code. The 4 individual Kv7.1 subunits are shown as ribbons and colored cyan, magenta, green and yellow. Docking of 15 Rottlerin molecules (blue) to the model structure of Kv7.1 (green, middle) or Kv7.1/KCNE1 (Kv7.1-green / KCNE1-yellow, bottom) in open states. Rottlerin molecules dock exclusively to outer regions of the channel model.

Rottlerin alters cardiac repolarisation in iPSC cardiomyocytes overexpressing Kv7.1/ KCNE1 channels

A human transgenic iPSC line over-expressing hKv7.1 was trans-differentiated to result in beating cardiac myocytes. The electrical activity was monitored by multi-electrode array technique. The extracellularly recorded local field potential is similarly to the human

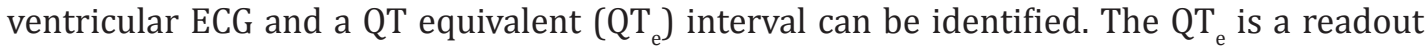
of cardiac cell repolarization. Rottlerin, applied at $2 \mu \mathrm{M}$ and $5 \mu \mathrm{M}$, evoke a significant and dose-dependent shortening of QT (Fig. 5). Thus, Rottlerin is effectively shortening cardiac repolarisation in human iPSC cardiomyocytes, presumably by activation of Kv7.1.

\section{Discussion}

There is a widely recognized need for improved therapies in $\mathrm{K}_{\mathrm{v}}$ 7-based channelopathies like LQTS, elevated blood pressure, diabetes, and hearing impairment [34]. A drug that 


\section{Cellular Physiology Cell Physiol Biochem 2016;40:1549-1558

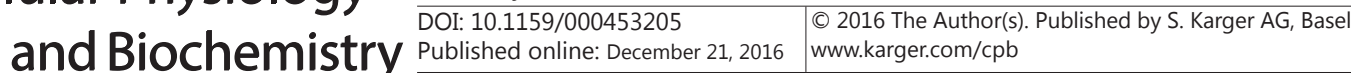 \\ Matschke et al.: Rottlerin Modulates Kv7 Channels}

Fig. 5. Functional effect of Rottlerin on Kv7.1/KCNE1 channels in human iPSC cardiomyocytes. $A$ IPSC cardiomyocytes expressing Kv7.1-EGFP were plated on MEA and the corrected QTe (QTc) was recorded under control conditions and after application of $2 \mu \mathrm{M}$ and $5 \mu \mathrm{M}$ Rottlerin. ( $\mathrm{n}=7, \pm$ SEM, paired t-test $\left.{ }^{*} \mathrm{p}<0.05\right)$. $B$ Example of one MEA experiment indicating the corrected maximal QT-equivalent (QTe-cmax) under basal conditions, at $2 \mu \mathrm{M}$ and $5 \mu \mathrm{M}$ Rottlerin after 4 or 10 min of Rottlerin application.

activates the respective Kv7 isoform might be useful for treatment of inherited forms of those disorders [1]. R-L3 has been the first $\mathrm{K}_{\mathrm{v}}$ 7.1/KCNE1 activator reported, which shortens action potential duration [19] and suppresses early after-depolarizations in ventricular myocytes isolated from canine hearts [21]. The effects of R-L3 on Kv7.1 and Kv7.1/KCNE1 channels are complex, leading to increased current magnitude, mildly slowed channel activation, but dramatically decreased rate of channel deactivation [20]. The more recently identified Kv7.1 and Kv7.4 activators have complex effects on the Kv7 channels [22-26], but often lack selectivity. Due to the need of and potential relevance as drug candidates, we searched for additional, new Kv7.1 and Kv7.4 activating compounds.

We identified Rottlerin as a potent Kv7.1 and Kv7.4 activator. Rottlerin is also called Mallotoxin. It is a natural polyphenolic compound isolated from the monkey-faced tree Mallotus phillippensis [35]. It has been reported to uncouple mitochondria [36]. The mitochondrial uncoupling depolarizes the mitochondrial membrane potential and influences mitochondrial production of reactive oxygen species (ROS) [37]. Furthermore, the compound reduces intracellular ATP levels and activates 5'-AMP-activated protein kinase (AMPK) [36]. It has also been reported as an inhibitor of PKC $\delta$ [38], whereas this effect may be indirect [35]. Rottlerin modulates asymmetry of plasma membrane by affecting cellular scramblase [39], and Rottlerin has been identified as a modulator of the BK and hERG $\mathrm{K}^{+}$channels [40, 41]. In the electrophysiological recordings presented here, Rottlerin was identified as a modulator of Kv7.1 and Kv7.4 channels heterologously expressed in Xenopus laevis oocytes. We could not identify significant Rottlerin sensitivity of Kv7.2, Kv7.2/Kv7.3 or Kv7.5 following application of $30 \mu \mathrm{M}$ (Fig. 1B, C).

In the heart and the inner ear, Kv7.1 channels associate with the ß-subunit KCNE1 [27, $42,43]$ which impacts on the pharmacological profile of Kv7.1 channel complexes [20, 28, 29]. The sensitivity of these heteromeric channels to Rottlerin was only slightly reduced $\left(\mathrm{EC}_{50}=4.9 \mu \mathrm{M}\right)$ compared to that of homomeric Kv7.1 (EC $\left.=1.48 \mu \mathrm{M}\right)$ (Fig. 2). These data identify Rottlerin as promising candidate to modulate Kv7.1 and/or Kv7.4 associated channelopathies.

The very high sensitivity of Kv7.4, as well as the moderate sensitivity of Kv7.1 and Kv7.1/KCNE1 suggests Rottlerin as a promising candidate as a scaffold for the generation of highly potent and selective Kv7.1 and/or Kv7.4 activators. In order to gain insights into Rottlerin binding to Kv7.1/KCNE1 channels, we tested Rottlerin effects in mutant channels with disrupted ubiquitin-guided endocytosis $\left(\mathrm{Kv} 7.1^{\Delta \mathrm{PY}}\right)[32]$ or with destroyed R-L3 binding site (Kv7.1 ${ }^{1337 v}$ ) [33]. Clearly, only the mutant with disrupted R-L3-activator binding site is insensitive to Rottlerin activation (Fig. 4A). These data suggest that Rottlerin may bind directly to the channel complex to activate it. In silico simulations and automatic docking of Rottlerin to a Kv7.1 channel model identifies several putative binding sites, which are localized outside the pore domain in proximity of the voltage sensor domain (Fig. 5B). This position is similar to the R-L3 binding site supporting the idea of direct binding to the R-L3 


\section{Cellular Physiology Cell Physiol Biochem 2016;40:1549-1558 \begin{tabular}{ll|l} 
DOI: 10.1159/000453205 & O) 2016 The Author(s). Published by S. Karger AG, Basel \\
and Biochemistry
\end{tabular} \\ Matschke et al.: Rottlerin Modulates Kv7 Channels}

site. De novo synthesis of the complex polyphenolic Rottlerin molecule has been reported recently [44]. Using such a total synthetic approach will potentially allow for selective modification of e.g. the hydroxyls within the Rottlerin scaffold, which can be tested and may allow for generation of selective compounds with novel and unique pharmacological profiles.

Currently, there is not a single selective Kv7.4 activator with a comparably high potency published. Especially, a high selectivity for Kv7.4 vs Kv7.2/Kv7.3 is desirable to prevent unintended side effects during acute pain processing [45]. Therefore, Rottlerin represents a promising candidate as a tool compound. As Kv7.4 is highly expressed in the inner ear and vestibular organs hair cells, Rottlerin represents a scaffold for generation of potential drug candidates for inner ear/vestibular diseases like tinnitus or vertigo. Further, the Rottlerin scaffold is potentially suitable to generate potent Kv7.1 selective drug candidates. Partial inhibition may be beneficial to treat short QT syndromes and reduce pathological over-activity of $\mathrm{I}_{\mathrm{Ks}}$ in patients with decreased risk of drug induced LQTS. On the other hand, activation of $\mathrm{I}_{\mathrm{Ks}}$ may be a strategy to recover function of $\mathrm{I}_{\mathrm{Ks}}$ in LQTS. Indeed, our experiments on iPSC cardiomyocytes clearly show that Rottlerin dose-dependently shortens cellular repolarisation. Therefore, Rottlerin or an optimized derivative holds a potential as QTinterval correcting drug.

\section{Abbreviations}

Rottlerin is 5,7-dihydroxy-2,2-dimethyl-6-(2,4,6-trihydroxy-3-methyl-5-acetylbenzyl)8-cinnamoyl-1,2-chromine.

\section{Acknowledgements}

This work was supported by grants from the Deutsche Forschungsgemeinschaft DFGSe1077/3-3, DFG INST 2105/27-1 to SGM and IMF Muenster.

\section{Disclosure Statement}

The authors declare that they have no conflict of interest.

\section{References}

1 Seebohm G: Activators of cation channels: Potential in treatment of channelopathies. Mol Pharmacol 2005;67:585-588.

2 Maljevic S, Wuttke TV, Seebohm G, Lerche H: Kv7 channelopathies. Pflugers Arch 2010;460:277-288.

3 Seebohm G: Kv7.1 in atrial fibrillation. Heart Rhythm 2009;6:1154-1155.

4 Wang Q, Curran ME, Splawski I, Burn TC, Millholland JM, VanRaay TJ, Shen J, Timothy KW, Vincent GM, de Jager T, Schwartz PJ, Toubin JA, Moss AJ, Atkinson DL, Landes GM, Connors TD, Keating MT: Positional cloning of a novel potassium channel gene: Kvlqt1 mutations cause cardiac arrhythmias. Nature Genet 1996;12:17-23.

5 Goldman AM, Glasscock E, Yoo J, Chen TT, Klassen TL, Noebels JL: Arrhythmia in heart and brain: Kcnq1 mutations link epilepsy and sudden unexplained death. Sci Transl Med 2009;1:2ra6.

6 Holmkvist J, Banasik K, Andersen G, Unoki H, Jensen TS, Pisinger C, Borch-Johnsen K, Sandbaek A, Lauritzen T, Brunak S, Maeda S, Hansen T, Pedersen O: The type 2 diabetes associated minor allele of rs2237895 kcnq1 associates with reduced insulin release following an oral glucose load. PloS one 2009; 4:e5872.

7 Yasuda K, Tanahashi H, Hayashi M, Yamakita N: Hyperglycemic crises in adult patients with diabetes: Response to kitabchi et al. Diabetes Care 2009;32:e157; author reply e158. 


\section{Cellular Physiology Cell Physiol Biochem 2016;40:1549-1558

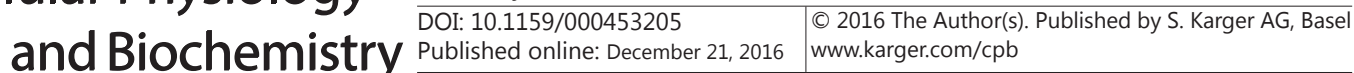 \\ Matschke et al.: Rottlerin Modulates Kv7 Channels}

8 Frohlich E, Engel E, Wahl R: Decrease in dipeptidyl peptidase iv activity is linked to the efficacy of differentiating compounds in follicular thyroid carcinoma cell lines. Horm Metabol Res 2011;43:364-366.

9 Roepke TK, King EC, Reyna-Neyra A, Paroder M, Purtell K, Koba W, Fine E, Lerner DJ, Carrasco N, Abbott GW: Kcne2 deletion uncovers its crucial role in thyroid hormone biosynthesis. Nature Med 2009;15:11861194.

10 Biervert C, Schroeder BC, Kubisch C, Berkovic SF, Propping P, Jentsch TJ, Steinlein OK: A potassium channel mutation in neonatal human epilepsy. Science 1998;279:403-406.

11 Charlier C, Singh NA, Ryan SG, Lewis TB, Reus BE, Leach RJ, Leppert M: A pore mutation in a novel kqt-like potassium channel gene in an idiopathic epilepsy family. Nature Genet 1998;18:53-55.

12 Singh NA, Charlier C, Stauffer D, DuPont BR, Leach RJ, Melis R, Ronen GM, Bjerre I, Quattlebaum T, Murphy JV, McHarg ML, Gagnon D, Rosales TO, Peiffer A, Anderson VE, Leppert M: A novel potassium channel gene, kcnq2, is mutated in an inherited epilepsy of newborns. Nature Genet 1998;18:25-29.

13 Jepps TA, Chadha PS, Davis AJ, Harhun MI, Cockerill GW, Olesen SP, Hansen RS, Greenwood IA: Downregulation of kv7.4 channel activity in primary and secondary hypertension. Circulation 2011;124:602-611.

14 Kubisch C, Schroeder BC, Friedrich T, Lutjohann B, El-Amraoui A, Marlin S, Petit C, Jentsch TJ: Kcnq4, a novel potassium channel expressed in sensory outer hair cells, is mutated in dominant deafness. Cell 1999;96:437-446.

15 Chouabe C, Neyroud N, Guicheney P, Lazdunski M, Romey G, Barhanin J: Properties of kvlqt1 k+ channel mutations in romano-ward and jervell and lange-nielsen inherited cardiac arrhythmias. EMBO J 1997; 16:5472-5479.

16 Lerche H, Biervert C, Alekov AK, Schleithoff L, Lindner M, Klinger W, Bretschneider F, Mitrovic N, JurkatRott K, Bode H, Lehmann-Horn F, Steinlein OK: A reduced k+ current due to a novel mutation in kcnq2 causes neonatal convulsions. Ann Neurol 1999;46:305-312.

17 Seebohm G, Scherer CR, Busch AE, Lerche C: Identification of specific pore residues mediating kcnq1 inactivation. A novel mechanism for long qt syndrome. J Biol Chem 2001;276:13600-13605.

18 Wollnik B, Schroeder BC, Kubisch C, Esperer HD, Wieacker P, Jentsch TJ: Pathophysiological mechanisms of dominant and recessive kvlqt1 $\mathrm{k}+$ channel mutations found in inherited cardiac arrhythmias. Human Mol Gen 1997;6:1943-1949.

19 Salata JJ, Jurkiewicz NK, Wang J, Evans BE, Orme HT, Sanguinetti MC: A novel benzodiazepine that activates cardiac slow delayed rectifier k+ currents. Molec Pharmacol 1998;54:220-230.

20 Seebohm G, Chen J, Strutz N, Culberson C, Lerche C, Sanguinetti MC: Molecular determinants of kcnq1 channel block by a benzodiazepine. Mol Pharmacol 2003;64:70-77.

21 Xu X, Salata JJ, Wang J, Wu Y, Yan GX, Liu T, Marinchak RA, Kowey PR: Increasing i(ks) corrects abnormal repolarization in rabbit models of acquired lqt2 and ventricular hypertrophy. Am J Physiol Heart Circul Physiol 2002;283:H664-670.

22 Mattmann ME, Yu H, Lin Z, Xu K, Huang X, Long S, Wu M, McManus OB, Engers DW, Le UM, Li M, Lindsley CW, Hopkins CR: Identification of (r)-n-(4-(4-methoxyphenyl)thiazol-2-yl)-1-tosylpiperidine2-carboxamide, ml277, as a novel, potent and selective $\mathrm{k}(\mathrm{v}) 7.1$ (kcnq1) potassium channel activator. Bioorgan Med Chem Let 2012;22:5936-5941.

23 Mruk K, Kobertz WR: Discovery of a novel activator of kcnq1-kcne1 k channel complexes. PloS one 2009;4:e4236.

24 Sun DD, Wang HC, Wang XB, Luo Y, Jin ZX, Li ZC, Li GR, Dong MQ: Tanshinone iia: A new activator of human cardiac kcnq1/kcne1 (i(ks)) potassium channels. Eur J Pharmacol 2008;590:317-321.

25 Zheng Y, Zhu X, Zhou P, Lan X, Xu H, Li M, Gao Z: Hexachlorophene is a potent kcnq1/kcne1 potassium channel activator which rescues lqts mutants. PloS one 2012;7:e51820.

26 Schroder RL, Strobaek D, Olesen SP, Christophersen P: Voltage-independent kcnq4 currents induced by (+/-)bms-204352. Pflugers Arch 2003;446:607-616.

27 Strutz-Seebohm N, Seebohm G, Fedorenko O, Baltaev R, Engel J, Knirsch M, Lang F: Functional coassembly of kcnq4 with kcne-beta- subunits in xenopus oocytes. Cell Physiol Biochem 2006;18:57-66.

28 Lerche C, Bruhova I, Lerche H, Steinmeyer K, Wei AD, Strutz-Seebohm N, Lang F, Busch AE, Zhorov BS, Seebohm G: Chromanol 293b binding in kcnq1 (kv7.1) channels involves electrostatic interactions with a potassium ion in the selectivity filter. Mol Pharmacol 2007;71:1503-1511. 


\section{Cellular Physiology Cell Physiol Biochem 2016;40:1549-1558 \begin{tabular}{ll|l} 
DOI: 10.1159/000453205 & $\begin{array}{l}\text { O 2016 The Author(s). Published by S. Karger AG, Basel } \\
\text { www.karger.com/cpb }\end{array}$
\end{tabular} \\ Matschke et al.: Rottlerin Modulates Kv7 Channels}

29 Lerche C, Seebohm G, Wagner CI, Scherer CR, Dehmelt L, Abitbol I, Gerlach U, Brendel J, Attali B, Busch AE: Molecular impact of mink on the enantiospecific block of i(ks) by chromanols. Br J Pharmacol 2000;131:1503-1506.

30 Henrion U, Strutz-Seebohm N, Duszenko M, Lang F, Seebohm G: Long qt syndrome-associated mutations in the voltage sensor of i(ks) channels. Cell Physiol Biochem 2009;24:11-16.

31 Zhang M, D'Aniello C, Verkerk AO, Wrobel E, Frank S, Ward-van Oostwaard D, Piccini I, Freund C, Rao J, Seebohm G, Atsma DE, Schulze-Bahr E, Mummery CL, Greber B, Bellin M: Recessive cardiac phenotypes in induced pluripotent stem cell models of jervell and lange-nielsen syndrome: Disease mechanisms and pharmacological rescue. Proc Natl Acad Sci USA 2014;111:E5383-5392.

32 Jespersen T, Membrez M, Nicolas CS, Pitard B, Staub O, Olesen SP, Baro I, Abriel H: The kcnq1 potassium channel is down-regulated by ubiquitylating enzymes of the nedd4/nedd4-like family. Cardiovasc Res 2007;74:64-74.

33 Seebohm G, Pusch M, Chen J, Sanguinetti MC: Pharmacological activation of normal and arrhythmiaassociated mutant kcnq1 potassium channels. Circul Res 2003;93:941-947.

34 Xiong Q, Sun H, Zhang Y, Nan F, Li M: Combinatorial augmentation of voltage-gated kcnq potassium channels by chemical openers. Proc Natl Acad Sci USA 2008;105:3128-3133.

35 Soltoff SP: Rottlerin: An inappropriate and ineffective inhibitor of pkcdelta. Trends Pharmacol Sci 2007;28:453-458.

36 Soltoff SP: Rottlerin is a mitochondrial uncoupler that decreases cellular atp levels and indirectly blocks protein kinase cdelta tyrosine phosphorylation. J Biol Chem 2001;276:37986-37992.

37 Izeradjene K, Douglas L, Tillman DM, Delaney AB, Houghton JA: Reactive oxygen species regulate caspase activation in tumor necrosis factor-related apoptosis-inducing ligand-resistant human colon carcinoma cell lines. Cancer Res 2005;65:7436-7445.

38 Gschwendt M, Muller HJ, Kielbassa K, Zang R, Kittstein W, Rincke G, Marks F: Rottlerin, a novel protein kinase inhibitor. Biochem Biophys Res Com 1994;199:93-98.

39 Frasch SC, Henson PM, Kailey JM, Richter DA, Janes MS, Fadok VA, Bratton DL: Regulation of phospholipid scramblase activity during apoptosis and cell activation by protein kinase cdelta. J Biol Chem 2000;275:23065-23073.

40 Zakharov SI, Morrow JP, Liu G, Yang L, Marx SO: Activation of the bk (slo1) potassium channel by mallotoxin. J Biol Chem 2005;280:30882-30887.

41 Zeng H, Lozinskaya IM, Lin Z, Willette RN, Brooks DP, Xu X: Mallotoxin is a novel human ether-a-go-gorelated gene (herg) potassium channel activator. J Pharmacol Exp Ther 2006;319:957-962.

42 Barhanin J, Lesage F, Guillemare E, Fink M, Lazdunski M, Romey G: K(v)lqt1 and lsk (mink) proteins associate to form the i(ks) cardiac potassium current. Nature 1996;384:78-80.

43 Sanguinetti MC, Curran ME, Zou A, Shen J, Spector PS, Atkinson DL, Keating MT: Coassembly of k(v)lqt1 and mink (isk) proteins to form cardiac i(ks) potassium channel. Nature 1996;384:80-83.

44 Hong KK, Ball GE, Black DS, Kumar N: The mosaic of rottlerin. J Org Chem 2015;80:10668-10674.

45 Cerina M, Szkudlarek HJ, Coulon P, Meuth P, Kanyshkova T, Nguyen XV, Göbel K, Seidenbecher T, Meuth SG, Pape HC, Budde T. Thalamic Kv 7 channels: pharmacological properties and activity control during noxious signal processing. Br J Pharmacol 2015;172:3126-3140. 